\title{
Proteins as biocatalysts and biomaterials
}

\author{
Nair V.K. ${ }^{1}$, More M.K. ${ }^{2}$, Sawant J.J. ${ }^{3}$, Thikekar V.P. ${ }^{4}$, Philip P.A. ${ }^{5}$, Ojha M.D. ${ }^{6}$, and Gomase V.S. ${ }^{7}$ \\ ${ }^{1}$ Bhavan's College, Andheri (W), Mumbai- 400058, India \\ ${ }^{2}$ Tilak College of Science and Commerce, Vashi, Navi Mumbai \\ ${ }^{3}$ Gogate Jogalekar College of Science, Arts and Commerce, Ratnagiri, India \\ ${ }^{4}$ Bhartiya Gramin Punarrachana Sanstha's Arts, Commerce and Science, Wadala,Mumbai, India \\ ${ }^{5}$ C.M.S. College of Science and Commerce, Tamil Nadu, India \\ ${ }^{6}$ I.C.L.E's Motilal Jhunjhunwala College of Arts, Science and Commerce, Vashi, Navi Mumbai \\ ${ }^{7}$ School of Technology, S.R.T.M. University, Sub-Centre, Latur, 413512, India
}

\begin{abstract}
This article reviews the available literature on the function of proteins as biocatalysts and biomaterials. It also explains methods like protein designing, enzyme evolution, metabolic pathway engineering and systems biology. Further, it deals with the discovery and development of extremophilic enzymes and industrial enzymes along with the case studies on novel economical industrial enzymes.

Keywords- Biocatalyst, Biomaterial, Protein Design, Extremophilic Enzyme, Protein Biomaterial Design
\end{abstract}

\section{Introduction}

Proteins are large, complex molecules which play many critical roles in the body. They do work in cells and are essential for the structure, function, and regulation of the body's tissues and organs. They are capable of carrying out variety of functions. Some of the examples of protein function are: Antibody, Enzyme, Messenger, Structural Component, Transport and Storage. Apart from these functions, proteins can also act as Biocatalysts and Biomaterials. Biocatalyst is a substance that initiates or modifies the rate of chemical reaction in a living body, i.e., a biochemical catalyst. They provide an economical and energy efficient tool for future industrial applications [29]. The requirements for a useful biocatalyst are selectivity, volume efficiency, security of supply and technology integration [33]. A Biomaterial is a synthetic or natural material used to replace part of a living system or to function in intimate contact with living tissue. Protein design is the design of new protein molecules or the deliberate design of a new molecule by introducing variations on a known structure. The rational design technique, which is used in protein engineering requires an understanding of the molecular interactions that stabilize proteins in specific folded configurations [5]. Metabolic Engineering is the purposeful alteration of metabolic pathways found in an organism to better understand and utilize cellular pathways for chemical transformation, energy transduction and supramolecular assembly. The systems biology approach involves the development of mechanistic models, like the reconstruction of dynamic systems from the quantitative properties of their elementary building blocks. The discovery and development of extremophilic enzymes is of utmost importance in industrial applications. The design of biomaterials that elicit specific cellular behavior constitutes a major challenge for the fields of tissue engineering and materials science [44].
Enzymes are proteins that increase the rates of (catalyze) chemical reactions. In enzymatic reactions, enzyme converts substrates into different molecules, called the products [22].

\section{Protein acts as biocatalysts}

Regioselectivity of glycosyltransferases offered an important means to overcome the limitations of chemical synthesis of small molecule glycosides. A large multigene family of UDPglucose: glycosyltransferases of Arabidopsis was explored for their potential as novel biocatalysts for in vitro synthesis and whole-cell catalysis. Quercetin was used as a substrate for this study because the flavonol and its glycosides has important medicinal properties and the metabolite provided a complex structure for regioselective glucosylation. The activity of 91 recombinant enzymes was analyzed for in vitro activity toward quercetin and discovered 29 that were capable of glucosylating the substrate. It was also shown that the regioselectivity of glucosylation could be maintained when the enzymes were used as whole-cell biocatalysts in Escherichia coli [1]. A crude extract rich in plant cysteine peptidases was obtained from the latex of the fruits of Araujia hortorum. The highly concentrated extract was immobilized onto titanium dioxide to produce biocatalysts through a simple adsorption procedure. Absorbance measurement at $280 \mathrm{~nm}$ and Bradford's method for protein quantification revealed that the protein content of the crude extract was selectively adsorbed onto the titanium dioxide surface at a very high rate. $\ln 5$ min of contact with the support all protein present in the crude extract was selectively withdrawn from the solution, leading to an immobilized biocatalyst with a high protein concentration [2]. Drug metabolizing mutants of cytochrome P450 BM3 (CYP102A1) can be used as biocatalysts for generation of reactive metabolites [28]. Biomaterials interface with biological systems to evaluate, treat, augment or replace any tissue, organ or function of the body. Biomaterials are 
mostly non-viable, but may also be viable. Silk biomaterials are used in: Joint replacements, Bone plates, Bone cement, Wound healing, Artificial ligaments and tendons, Dental implants for tooth fixation, Blood vessel prostheses, Heart valves, Skin repair devices, Cochlear replacements, Contact lenses[26].

\section{Proteins acts as biomaterial}

Fibroblast growth factor (FGF-2) derived proteins were able to accelerate the endothelialization of medical prostheses and the reendothelialization of injured blood vessels and generating an endothelium with macroscopic characteristics which was similar to a healthy endothelium and also minimizing restenosis of medical prostheses or blood vessels [3]. Flagelliform silk was introduced to describe a general principle for designing genes of repetitive performance proteins for recombinant expression in Escherichia coli. In the first step, repeating amino acid sequence motifs were reversely transcripted into DNA cassettes, which could in a second step, is seamlessly ligated to yield a designed gene. Recombinant expression led to proteins mimicking the natural ones. The recombinant proteins could be assembled into nanostructured materials in a controlled manner thus allowing their use in several applications [4].

\section{Protein Design}

Protein design can also be referred to as inverse folding. Designing a new protein involves the identification of the sequences which have chosen structure as free energy minimum. This can be done by use of computer models, which generate sequences to fold on the desired structure [5]. The ability to specify and design artificial proteins opens the way to understand its structure, stability and folding kinetics to accomplish de novo protein design. Directed evolution coupled with high-throughput robotic screen was employed to broaden the industrial use of enzymes [36]. Protein engineering is used in designing tailored enzymes and microorganisms for biofuel production. Computational protein design algorithms seek to identify amino acid sequences with low energies for the target structures. The most challenging requirement for computational protein design is a fast, but accurate, energy function which can distinguish optimal sequences from similar suboptimal ones. Using computational methods, a protein with a novel fold can be designed [5]. The protein design methodology, applied to the complete redesign of 27 proteins was tested and study on how the entropy affects the ranking of the same sets of sequences at low and high temperatures and how the new methodology affects the fraction of aminoacids of each kind that were found in solvent-protected positions was done. Results indicated that accounting for entropic contribution in the score function affected the outcome in a highly non-trivial way, and might improve current computational design techniques based on protein stability. Indeed, ranking at low and high temperatures were, weakly correlated, which pointed out the importance of accounting for the entropy. The free-energy driven design yielded sequences that differred in many positions from those obtained with the standard design, while the burial fraction for the aminoacids did not change much [6]. A quantitative design algorithm based on the physical properties that determine protein structure and stability, which is not limited to specific folds or motifs was developed. This design developed methods for residues of all parts of a protein. The experimental characterization of pda8d showed that, it was folded and well-ordered with a weakly cooperative thermal transition, and that its structure was an excellent match to the design target. Pda8d is the shortest sequence of naturally occurring amino acids that folded to a unique structure without metal binding, oligomerization or disulfide bond formation. The successful design of pda8d supported the use of objective, quantitative sequence selection algorithms for protein design. This robustness suggested that the algorithm can be used to design sequences for de novo backbones [7]. A 'protein design cycle' led to recent advances in rational protein design. An approach, in which protein positions were classified by their local environments, aided development of an appropriate energy expression. The design of proteins that folded to a specified target backbone structure was becoming possible. Discovering the forces critical to the determination of backbone conformation and their coupling to sequence selection rised as the major challenge in solving the 'complete' protein design problem. A general ability to design specific protein structures paved the way towards the goal of rationally designing novel functional molecules [8]. Two distinct and yet complementary protein design approaches, rational design and directed evolution, has been successfully developed to engineer biocatalysts with altered or novel stability, activity, substrate specificity, selectivity, cofactor specificity, reaction chemistry, and $\mathrm{pH}$ optima. Recently, a third approach combining the best of rational design and directed evolution had also shown great promise in protein engineering, which would attract increasing attention in the near future [9].

\section{Enzymes Evolution}

Enzyme evolution studies provide strong evidence that nucleic acid polymerases have a common ancestor. It has been postulated that most of the nucleic acid polymerase belong to a 
polymerase superfamily that contain closely related active sites that are similarly positioned within the polymerase active cleft. Thus, it appears that there are genetic polymerase modules that provide the active site to carry out the phosphoryl transfer reaction. Only subtle modifications achieve the substrate specificity that is unique for each polymerase class. We see in the course of evolution the tendency of nature to copy and paste particular structural motifs. Evolution chose enzymes to be proteins and not carbohydrates. Engineering the specificity and properties of enzymes and proteins within rapid time frames became feasible with the advent of directed evolution. New functions were engineered by introducing and recombining mutations, followed by subsequent testing of each variant for the desired new function. A range of available methods for mutagenesis were used to introduce mutations at single sites, targeted regions within a gene or randomly throughout the entire gene. Currently, enzyme engineers are still searching which combinations of selection methods and techniques for mutagenesis and DNA recombination are most efficient. These techniques were successfully used for the creation of novel proteins for biocatalysis and the life sciences [10]. Several models have been proposed to explain the origin and evolution of enzymes in metabolic pathways. Initially, the retro-evolution model proposed that, as enzymes at the end of pathways depleted their substrates, there was a pressure for earlier enzymes in pathways to be created, using the later ones as initial template, in order to replenish the pools of depleted metabolites. Later, the recruitment model proposed that initial templates from other pathways could be used as long as those enzymes were similar in chemistry or substrate specificity. A network approach was used to study enzyme evolution in fully sequenced genomes, thus removing both constraints. It was found that homologous pairs of enzymes were roughly twice as likely to have evolved from enzymes that were less than three steps away from each other in the reaction network than pairs of non-homologous enzymes. These results, together with the conservation of the type of chemical reaction catalyzed by evolutionarily related enzymes, suggested that functional blocks of similar chemistry have evolved within metabolic networks. One possible explanation for these observations is that this local evolution phenomenon is likely to cause less global physiological disruptions in metabolism than evolution of enzymes from other enzymes that are distant from them in the metabolic network [11]. In order to clarify the status of the malate synthase (MS) and isocitrate lyase (ICL) genes in animals and get an insight into their evolution, a comparative-genomic study was undertaken.
Using sequence similarity searches, MS genes were identified in arthropods, echinoderms, and vertebrates, including platypus and opossum, but not in the numerous sequenced genomes of placental mammal's. The regions of the placental mammals' genomes expected to code for malate synthase showed clear similarity to the opossum MS sequence but contained stop codons, indicating that the MS gene became a pseudogene in placental mammals. By contrast, the ICL gene was undetectable in animals other than the nematodes that possessed a bifunctional, fused ICL-MS gene. Examination of phylogenetic trees of MS and ICL suggested multiple horizontal gene transfer events that probably went in both directions between several bacterial and eukaryotic lineages. The strongest evidence was obtained for the acquisition of the bifunctional ICL-MS gene from unknown bacterial source with the corresponding operonic organization by the common ancestor of the nematodes. Distribution of the MS and ICL genes in animals suggested that either they encode alternative enzymes of the glyoxylate cycle that are not orthologous to the known MS and ICL or the animal MS acquired a new function that remains to be characterized. The genes for the glyoxylate cycle enzymes present a remarkable variety of evolutionary events including unusual horizontal gene transfer from bacteria to animals [12].

\section{Metabolic Pathway Engineering and Systems Biology}

An emerging approach to understand and utilize metabolic processes is Metabolic (or pathway) Engineering (ME). ME involves the redirection of cellular activities by the rearrangement of the transport, enzymatic, and regulatory functions of the cell by the use of recombinant DNA and other techniques. This can then be used to modify biological pathways for production of chemicals to improve plant productivity and safer energy production and study of medical conditions. Using this approach, industrial E.coli strain that produces 1, 3- propanediol at very high yields was successfully developed and it was also used to increase production of ascorbic acid intermediates [34,35]. Recent examples of metabolic engineering illustrate different strategies for the production of secondary metabolites in plants and microbial cells [38-41]. The term Systems Biology describes the field of scientific inquiry which takes a global approach to the understanding of cells and also elucidation of biological processes and mechanisms [13]. Systems biology is a biology-based interdisciplinary study field which focuses mainly on the systematic study of complex interactions in biological systems. One of the goals of systems biology is to discover new emergent properties so that the processes happening in a biological 
system can be better understood. Due to the large number of parameters and variables in cellular networks, numerical and computational techniques are also used. These include new forms of computational model like the integration of information from the literature, using techniques of information extraction and text mining and the development of online databases and repositories for sharing data and models. System Biology has the ability to obtain, integrate and analyze complex data from multiple experimental sources by making use of interdisciplinary tools. Some typical technology platforms are: Transcriptomics, Proteomics, Metabolomics, Glycomics, Lipidomics, Interactomics, Fluxomics, Biomics [42]. Availability of the complete yeast genomic sequence paved the way for the development of new techniques such as the gene chips, i.e., DNA fragment microarrays or oligonucleotide chips, which enabled genome-wide expression monitoring. A genomic expression pattern obtained when $S$. cerevisiae was undergoing the metabolic shift from fermentation to respiration conformed to the known regulatory response of yeast, which illustrated the potential of the DNA microarray; additionally, a number of previously unknown responses were also identified. The gene chips clearly generated a vast amount of biological information concerning the yeast model system in the future, and this may also be used for further understanding of higher eukaryotic cells like human cells. Within a very short time frame, the expression patterns obtained from the gene chip technology had an exploratory function in gene regulation, and hence transcriptional activators and repressors may also be identified, which may help to define appropriate strategies for metabolic engineering. Extensive information about new "protein pathways" i.e., protein interactions such as signaling transduction pathways, also serves to identify potential targets for gene amplification or gene deletion. $S$. cerevisiae has a great potential for pathway engineering [14]. Metabolic pathway engineering can also be used for enhanced biohydrogen production [43]. Salt tolerant cyanobacteria share many cellular characteristics with higher plants and therefore are an ideal model system used for studying salinity stress. Here, the response of fully adapted Synechocystis sp. PCC6803 cells to the addition of $6 \% \mathrm{w} / \mathrm{v} \mathrm{NaCl}$ was investigated using proteomics combined with targeted analysis of transcripts. Synechocystis $s p$. PCC6803 has the ability to regulate essential metabolic processes to enable survival in high salt environments. This adaptation strategy was assisted by further regulation of proteins involved in non-metabolic cellular processes, supported by transcriptional and post-transcriptional control. Synechocystis cells adapted to high salt (6\%) over an acclimation period of 9 days. This study demonstrated the effectiveness of using a systems biology approach in answering environmental, and in particular, salt adaptation questions in Synechocystis sp. PCC6803 [15]. A web server called FMM (From Metabolite to Metabolite) was developed for its use in synthetic biology. FMM could reconstruct metabolic pathways from one metabolite to another metabolite among different species, based mainly on the Kyoto Encyclopedia of Genes and Genomes (KEGG) database and other integrated biological databases. Both local and global graphical views of the metabolic pathways were designed. FMM is an effective tool for applications in synthetic biology to produce both drugs and biofuels. FMM helps in metabolic engineering by reconstructing metabolic pathways for producing some valuable metabolites or secondary metabolites in bacteria or yeast, which has potential in drug production. Moreover, FMM can be used to compare the metabolic pathways between numerous species and connect metabolic pathways of different KEGG maps, suggesting that FMM is not only an effective tool for synthetic biology, such as in the production of drugs and biofuels, but also a useful resource for investigation in metabolism [16].

\section{Discovery and Development of Extremophilic Enzyme}

The industrial application of enzymes that can withstand extreme conditions greatly increased over the past decade. This is mainly a result of the discovery of novel enzymes from extremophilic microorganisms. Enzymes from thermophilic organisms have found the most practical commercial use to date because of their overall inherent stability. This has also led to a greater understanding of stability factors that are involved in adaptation of these enzymes to their unusual stressful environments. Extremophilic enzymes offer versatile tools for developments in a variety of industrial application and as novel biocatalysts [30].

\section{Discovery and development of industrial enzymes}

Glycosyl hydrolases can be used in applications ranging from baking and brewing, to bioenergy, to oil or gas recovery. Biocatalysts for these applications often required stability and functional activity at elevated temperatures. As such, heterotrophic hyperthermophilic microorganisms capable of using a variety of carbohydrates as carbon and energy sources were examined as sources of thermally stable enzymes. The biochemical and functional attributes of the glycosyl hydrolases from these and other organisms can often are inferred from primary amino acid sequence information. The current challenges are to fully understand the known 
glycosyl hydrolases by biochemical and biophysical investigations, and to identify and characterize glycosyl hydrolases not yet annotated within genome sequences. Using a functional genomics approach, combined with bioinformatic techniques and biochemical characterization, the genome of the hyperthermophilic archaeon Pyrococcus furiosus was probed to identify new glycosyl hydrolases. The open reading frame (ORF) PF0870 was identified by bioinformatic analysis to encode a glycosyl hydrolase that was found to hydrolyze pNP-alpha-maltopyranoside and maltotriose, thereby defining it as a novel b-amylase. Transcriptional response of $P$. furiosus grown on pullulan, starch, glycogen, maltose, cellobiose, trehalose, and maltose/cellobiose suggested an alternative biochemical role for a previously characterized exo-alpha-glucanase, the native form of which had been previously purified from $P$. furiosus cell extracts. The broad substrate specificity and high temperature optimum makes this an especially appealing enzyme for the final hydrolysis step in bioethanol production. The result demonstrates how functional genomics approaches combined with bioinformatics analysis and biochemical characterization can be utilized to determine the role of proteins encoded in unannotated ORFs of genome sequences [17]. Current advances in metagenomics have revolutionized the research in fields of microbial ecology and biotechnology, enabling the highthroughput discovery of new enzymes for industrial bioconversions. Extremophilic organisms are the least well understood because of our inability to isolate pure cultures. There are two obstacles for reaping the fruit of the microbial diversity of extremophiles: first, in spite of the recent progress in development of new culturing techniques most extremophiles cannot be cultured using traditional culturing technologies; and second, the problem of the very low biomass densities often occurs under the conditions hostile for life, which do not yield enough DNA and reduces the effectiveness of cloning [18].

Protein Biomaterial Design and Implications

Synthetic polymers provide a wide array of physical properties and chemical compositions that can be exploited as components in prosthetics, devices, and tissue-engineered constructs. This requires elucidating the biological response to biomaterials and the relationship between surface characteristics and cellular behavior. Lymphocytes that transiently appear at implant sites were observed clinically to be reactive. However, the role of lymphocytes in the tissue response and the mechanisms involved are still not clear. A research to gain insight into the interactions between lymphocytes and macrophages at biomaterial surfaces and explore the relationship between surface properties and lymphocyte/macrophage interactions was carried out. The studies addressed the hypothesis that biomaterial surface chemistries modulate the direct and indirect lymphocyte interactions with macrophages and foreign body giant cells. PETbased photograft copolymerized surfaces with hydrophobic, hydrophilic/neutral, hydrophilic/anionic, and hydrophilic/cationic characteristics were utilized as model surfaces. Quantification of select inflammatory mediators showed that hydrophilic/neutral and hydrophilic/anionic surfaces promoted proinflammatory responses and a reduced potential for ECM degradation relative to other surfaces. Researchers subsequently showed the capability of lymphocytes, through direct and indirect mechanisms, to enhance macrophage activation and production of pro-inflammatory mediators. Hydrophilic/neutral and hydrophilic/anionic surfaces were shown to be highly but distinctly activating. These surfaces also promoted distinct lymphocyte subset adhesion to macrophages and FBGCs compared to hydrophobic PET suggesting induction of differential macrophage and FBGC phenotypes on varying biomaterial surfaces. Direct cell-cell interactions enhanced IFN- $y$ production more so on the hydrophilic/anionic surfaces than on any other surface, indicating this surface as highly lymphocyte activating. These findings provided insight into lymphocyte and macrophage interactions in response to biomaterial surfaces along with evidence consistent with the hypothesis that distinct surface chemistries modulate lymphocyte and macrophage interactions. Finally, a mechanistic model which would provide a tool for further analysis of lymphocyte and macrophage interactions in response to biomaterial surfaces and a step toward quantitative predictability of biomaterialdependent processes was presented [19]. Combinatorial techniques also help in protein biomaterial design [25]. Biocompatible and biodegradable biomaterials were used to develop biological matrix or scaffolds not only for Tissue Engineering but also for various biomedical applications including, wound dressings, membrane filters and drug delivery. Silk nanofibers were fabricated and characterized for bone Tissue Engineering application. Two types of silk protein viz., natural cocoon fibers from silkworm Bombyx mori and transgenic spider silk from Nephila clavipes were evaluated for the feasibility of generating nanofibers using electrospinning procedure. The mechanical properties of silk nanofibers were tailored using various post-spinning methods. Nanocomposite nanofibers made up of silk and carbon nanotube (CNT) were fabricated with the intension of improving mechanical and electrical properties of the scaffolds. The biocompatibility of silk 
nanofiber scaffolds was compared with the nanofibrous scaffolds made up of Type I collagen and poly lactic-co-glycolic acid (PLAGA). The structural changes in silk protein in silkworm's glands during natural spinning process and the electrospinning process were studied using molecular dynamics simulation. Cell-scaffolds interaction study was carried out on various scaffolds using primary fetal bovine osteoblasts [20]. The non-linear spectroscopic imaging was used as a non-invasive method to characterize the morphological and structural aspects of silkworm silk fibroin-based biomaterials. Researchers demonstrated that two photon excited fluorescence and second harmonic generation were sensitive to the hydration, overall $\beta$ sheet content and molecular orientation of the sample. This approaches offered promise for identifying important connections between biomaterial design and functional engineered tissue development. The strategies described had broader implications for understanding and tracking the remodeling of degradable biomaterials under dynamic conditions both in vitro and in vivo. The non-linear imaging and spectroscopy approaches served as important tools for assessing and monitoring silk-based and potentially other, biomaterial properties that may be particularly useful in the context of tissue engineering applications [21]. Fibrous proteins and biomaterials based on elastin and elastin derived molecules have broad impact in protein design [31,32].

\section{Case Studies on Novel Economical Industrial Enzymes}

Almost all processes in a biological cell need enzymes at significant rates. Since enzymes are selective for their substrates and catalyze only a few reactions from among many possibilities, the set of enzymes made in a cell determines which metabolic pathways occur in that cell. They are used in the chemical industry and other industrial applications when extremely specific catalysts are required. However, enzymes in general are limited in the number of reactions they catalyze and also by their lack of stability in organic solvents and at high temperatures. Consequently, protein engineering is an active area of research and attempts to create new enzymes with novel properties, either by rational design or in vitro evolution. These efforts have begun to be successful, and a few enzymes have now been designed "from scratch" to speed up reactions that do not occur in nature $[22,45,46]$. The effective catalytic properties of industrial enzymes are used to aid reactions of mass quantities of chemicals [27]. Approaches like screening of biodiversity, genomic sequencing, directed evolution and phage display can be used for discovering novel industrial enzymes [37].
A specialised phage display method was developed to isolate extracellular proteins by virtue of the presence of signal peptides (SS phage display). It was successfully applied to $S$. aureus and, on a larger scale, to the symbiotic bacterium Bradyrhizobium japonicum. An inducible antisense RNA system was incorporated to enable gene silencing of the isolated genes. A tetracycline-regulated promoter was inserted in such a way, that an antisense RNA covering the cloned gene could be expressed. The new element was shown to be compatible with the properties of SS phage display, and to promote gene expression upon induction on both the transcriptional and translational level [23]. The development of home grown technologies for enzyme production and applications became one of the national priorities in industrial biotechnology. Enzyme production from indigenous microbial isolates were performed either by submerged or solid state fermentation processes. Based on its wide and unique spectrum of properties, enzymes have been developed for wide applications in various industrial processes. The development of the enzyme catalyzed applications is based on the modification of the reaction systems that enhances their catalytic activities. Some of the applications of the industrial enzymes include the fine chemicals production, oleochemicals modification, and detergent formulation, enzymatic deinking of waste papers, animal feed formulation and effluent treatment processes. Enzymes were also successfully used as analytical tool in the determination of compounds in body fluids. Although, most of these enzyme catalysed reactions were performed in aqueous phase, the use of enzymes in organic solvents was found to be significant for the production of new chemicals [24].

\section{References}

[1] Lim E.K., Ashford D.A., Hou B., Jackson R.G.,Bowles D.J. (2004) Biotechnology and bioengineering, 87 (5), 623-631.

[2] Llerena-Suster C.R., Foresti M.L., Briand L.E., Morcelle S.R. (2009) Colloids Surf B Biointerfaces, 72(1):16-24.

[3] Prats H., Laurell H., Bossard C., Garmy S., Barbara., Arnal.(2004) European patent application, EP20030290305.

[4] Vendrely C., Ackerschott C., Römer L., Scheibel T. (2008) Methods in molecular biology, 474, p.3-14.

[5] Saghatelian A., Yokobayashi Y., Soltani K., Reza M. (2001) Nature 409 (6822): 797-801.

[6] Daniele S., Pierpaolo B., Alessandro P., Marco P., Alfonso J. (2008) AIP Conference Proceedings.

[7] Dahiyat B. I., Sarisky C. A., Mayo S. L. (1997) J. Mol. Biol. 273, 789 \pm 796 . 
[8] Street A.G. and Mayo S.L.(1999) Structure, 7(5), R105-R109.

[9] Edward B.F. and Harbury P.B. (2007) Current Opinion in Structural Biology, 17:199-204

[10] Williams G.J., Nelson A.S., Berry A. (2004) Cell Mol Life Sci.;61(24):3034-46.

[11] Alves R., Chaleil R.A., Sternberg M.J. (2002) J Mol Biol, 22;324(2):387.

[12] Kondrashov F.A., Koonin E.V., Morgunov I.G., Finogenova T.V., Kondrashova M.N. (2006) Biol Direct. 23;1:31..

[13] Isidore R., Gregory S. Systems Biology, Volume I: Genomics, Oxford University Press, USA, 2006, ISBN-13: 9780195300819

[14] Ostergaard S., Olsson L., and Nielsen J. (2000) Microbiol Mol Biol, 64(1): 34-50.

[15] Pandhal J., Noirel J., Wright P.C., Biggs C.A. (2009) Saline Systems, 7;5:8.

[16] Chou C.H., Chang W.C., Chiu C.M., Huang C.C., Huang H.D. (2009) Nucleic Acids Res. 1;37(Web Server issue):W129-34.

[17] Chou Chung-Jung, Shockley Keith R., Conners Shannon B., Lewis Derrick L., Comfort Donald A., Adams Michael W. W., Kelly Robert M. (2007) Applied and environmental 73(21):6842-53.

[18] Ferrer M., Golyshina O., Beloqui A., Golyshin P.N. (2007) Current Opinion in Microbiology, 10 (3), 207-214.

[19] Chang D.T., Jones J.A., Meyerson H., Colton E., Kwon I.K., Matsuda T., Anderson J.M.(2008) J Biomed Mater Res A., 87(3):676-87.

[20] Li M., Mondrinos M.J., Chen X., Gandhi M.R., Ko F.K., Lelkes P.I. (2006) J Biomed Mater Res A.,79(4):963-73.

[21] Rice, William L., Firdous S., Gupta S., Hunter M., Foo Cheryl W. P.,Wang Y., Kim H. J. (2008) Biomaterials,29 (13), 2015-2024.

[22] Jaeger K.E., Eggert T. (2004) Curr Opin Biotechnol. 15 (4): 305-13

[23] Bjerketorp J., Rosander A., Nilsson M., Jacobsson K., Frykberg L.(2004) J Med Microbiol., 53(Pt 10):945-51.

[24] Ibrahim C. O. (2008) Bioresource technology, 99 (11), 4572-4582.

[25] Wingkono Gracy A.(2004), Georgia Institute of Technology.

[26] Vepari C., Kaplan D.L.(2007) Progress in Polymer Science, 32 (8), p.991-1007.

[27] Kirk O., Borchert T.V., Fuglsang C.C. (2002) Curr Opin Biotechnol. ;13(4):34551.

[28] Damsten M.C., van Vugt-Lussenburg B.M., Zeldenthuis T., Vlieger J.S., Commandeur J.N., Vermeulen N.P. (2008) Chem Biol Interact, 96-107.
[29] Chaput J.C., Woodbury N.W., Stearns L.A, Williams B.A. (2008) Expert Opin Biol Therapy, 1087-98.

[30] Antranikian G., Vorgias C.E., Bertoldo C. (2005) Adv Biochem Eng Biotechnol, 219-62.

[31] Wang X., Kim H.J., Wong C., Vepari C., Matsumoto A., Kaplan D. L. (2006) Materials Today, 9 (12), 44-53.

[32] Daamen Willeke F., Nillesen Suzan T. M., Wismans Ronnie G., Reinhardt Dieter P., Hafmans Theo., Veerkamp Jacques H., van Kuppevelt Toin H. (2008) Tissue engineering, 14(3):349-60.

[33] Taylor I.N., Brown R.C., Bycroft M., King G., Littlechild J.A., Lloyd M.C., Praquin C., Toogood H.S., Taylor S.J. (2004) Biochemical Society transactions, $32(\mathrm{Pt}$ 2), 290-292.

[34] Meynial S., Cervin I., Soucaille M. P. (2005) Applied and Environmental Microbiology, 2140-2144.

[35] Do-Young Yum, Bong-Yong Lee, DaeHyum Hahm, and Jae-Gu Pan (1998) J Bacteriol., 180(22): 5984-5988.

[36] Jones A., Lamsa M., Frandsen T.P., Spendler T., Harris P., Sloma A., Xu F., Nielsen J.B., Cherry, J.R. (2008) Journal of Biotechnology, 134 (3-4), 325-333.

[37] Marrs B., Delagrave S., Murphy D. (1999) Current Opinion in Microbiology, 241245.

[38] Mijts B. N. and Dannert C. S. (2003) Current Opinion in Biotechnology, 597602.

[39] Verpoorte R., Heijden R., Hoopen H.J.G., Memelink J. (1999) Biotechnology Letters, 467-479.

[40] Gunnarsson N., Eliasson A., Nielsen J.(2004) Adv Biochem Eng Biotechnol., 88:137-78.

[41] Nielsen J. (1998) Current opinion in microbiology, 330-336.

[42] Hiroaki K. (2002) Science , 295 (5560), 1662 - 1664.

[43] Mathews J., Wang G. (2009) International Journal of Hydrogen Energy, 74047416.

[44] Maskarinec S.A., Tirrell D. A. (2005) Current Opinion in Biotechnology, 16:422-426.

[45] Warshel A., Sharma P.K., Kato M., Xiang Y., Liu H., Olsson M.H. (2006) Chem. Rev. 106 (8): 3210-35.

[46] Olsson M.H., Parson W.W., Warshel A. (2006) Chem. Rev. 106 (5): 1737-56. 